\title{
Parameters of the SLAC Next Linear Collider ${ }^{*}$
}

\author{
T. Raubenheimer, C. Adolphsen, D. Burke, P. Chen, S. Ecklund, J. Irwin, G. Loew, \\ T. Markiewicz, R. Miller, E. Paterson, N. Phinney, M. Ross, R. Ruth, J. Sheppard, \\ H. Tang, K.Thompson \\ Stanford Linear Accelerator Center, Stanford University, Stanford, CA 94309 USA
}

Abstract

In this paper, we present the parameters and layout of the Next Linear Collider (NLC). The NLC is the SLAC design of a future linear collider using $\mathrm{X}$-band $\mathrm{RF}$ technology in the main linacs. The collider would have an initial centerof-mass energy of $0.5 \mathrm{TeV}$ which would be upgraded to 1 $\mathrm{TeV}$ and then $1.5 \mathrm{TeV}$ in two stages. The design luminosity is $>5 \times 10^{33} \mathrm{~cm}^{-2} \mathrm{sec}^{-1}$ at $0.5 \mathrm{TeV}$ and $>10^{34} \mathrm{~cm}^{-2} \mathrm{sec}^{-1}$ at 1.0 and $1.5 \mathrm{TeV}$. We will briefly describe the components of the collider and the proposed energy upgrade scenario.

\section{INTRODUCTION}

A number of groups around the world are creating designs for a future linear collider. The present state of the designs can be found in Ref. [1] and a summary of the present status and the required $R \& D$ can be found in Ref. [2]. In this paper, we describe the SLAC Next Linear Collider (NLC). The NLC would have an initial center-ofmass energy of $0.5 \mathrm{TeV}$ and would then be upgraded to 1 $\mathrm{TeV}$ and finally $1.5 \mathrm{TeV}$ in the center-of-mass.

The primary parameters for the three stages of the design are listed in Table 1. The parameters of the $500 \mathrm{GeV}$ collider are based upon technology that has been demonstrated or is expected to be demonstrated within the next few years. The upgrade path to $1 \mathrm{TeV}$ involves a very straightforward extrapolation of the RF technology, which could be expected to be ready by the time the collider starts operating at $500 \mathrm{GeV}$. Specifically, it requires that each of the $50 \mathrm{MW}$ klystrons be replaced with two $72 \mathrm{MW}$ klystrons. It also requires increasing the linac and final focus lengths by roughly $20 \%$. This additional length could be built into the $500 \mathrm{GeV}$ collider allowing the $1 \mathrm{TeV}$ energy upgrade to be made adiabatically by simply replacing and adding klystrons and modulators and replacing spool pieces at the end of the accelerator with accelerating structures.

At this time, there are many possible upgrade paths to $1.5 \mathrm{TeV}$. The $1.5 \mathrm{TeV}$ design will require further upgrades of the RF system to limit the AC power consumption. Examples are a Two Beam Accelerator concept from LBL and LLNL, grid-switched and cluster klystrons, and binary pulse compressors. In Table 1, we have listed a set of parameters which assumes a binary pulse compression system. To ensure both the possibility of the $1.5 \mathrm{TeV}$ upgrade and to provide operational flexibility, we are designing the primary components of the collider to allow for a substantial variation in parameters such as beam charge, accelerating gradient, etc. In the next sections, we will outline the components of the design and briefly summarize the $R \& D$

-Work supported by the Department of Energy, contract DE-AC03$76 \mathrm{SF} 00515$
Table 1. Parameters of NLC designs.

\begin{tabular}{|c|c|c|c|}
\hline CM Energy [TeV] & 0.5 & 1.0 & 1.5 \\
\hline Luminosity $\left[10^{33}\right]$ & 7.1 & 14.5 & 16.1 \\
\hline Rep. Rate [Hz] & 180 & 120 & 120 \\
\hline Bunch Charge $\left[10^{10}\right]$ & 0.7 & 1.1 & 1.1 \\
\hline Bunches/RF Pulse & 90 & 75 & 75 \\
\hline Bunch Sep. [ns] & 1.4 & 1.4 & 1.4 \\
\hline$\gamma \epsilon_{x} / \gamma \epsilon_{y}$ IP $\left[10^{-8} \mathrm{~m}-\mathrm{rad}\right]$ & $500 / 5$ & $500 / 5$ & $500 / 5$ \\
\hline$\beta_{x} / \beta_{y}$ IP $[\mathrm{mm}]$ & $10 / 0.1$ & $25 / 0.1$ & $37 / 0.15$ \\
\hline$\sigma_{x} / \sigma_{y}$ IP $[\mathrm{nm}]$ & $320 / 3.2$ & $360 / 2.3$ & $360 / 2.3$ \\
\hline$\sigma_{z}$ IP $[\mu \mathrm{m}]$ & 100 & 100 & 100 \\
\hline Upsilon & 0.09 & 0.27 & 0.41 \\
\hline Pinch Enhancement & 1.3 & 1.4 & 1.5 \\
\hline Beamstrahlung $\delta_{B}[\%]$ & 2.3 & 7 & 9 \\
\hline \# Photons per $e^{-} / e^{+}$ & 0.8 & 1.1 & 1.1 \\
\hline Loaded Gradient [MV/m] & 37 & 63 & 63 \\
\hline Active Linac Length $[\mathrm{km}]$ & 14.2 & 17.0 & 25.5 \\
\hline Total Site Length $[\mathrm{km}]$ & 20.0 & 25.5 & 36.2 \\
\hline \# of Klystrons & 3940 & 9456 & 7092 \\
\hline Klyst. Peak Pwr. [MW] & 50 & 72 & 76 \\
\hline Pulse Comp. Gain & 3.6 & 3.6 & 6.8 \\
\hline Power/Beam [MW] & 4.2 & 7.9 & 11.9 \\
\hline AC Power $[\mathrm{MW}]$ & 103 & 202 & 240 \\
\hline
\end{tabular}

status.

\section{II. $e^{+} / e^{-}$SOURCES}

The design of the NLC polarized electron source is based upon the Stanford Linear Collider (SLC) polarized source [3]. The SLC source very reliably delivers highly polarized ( $>80 \%$ ) beams to a damping ring at $1.2 \mathrm{GeV}$ with approximately $5 \times 10^{10}$ in a single bunch, a beam emittance of roughly $\gamma \epsilon_{x, y}=1 \times 10^{-4} \mathrm{~m}-\mathrm{rad}$, and an energy spread of $\pm 1 \%$. In the NLC design, the polarized electrons originate at a strained GaAs cathode DC biased at $-120 \mathrm{kV}$. To create the bunch train, the drive laser is sinusoidally modulated so that it delivers a pulse train of ninety $700 \mathrm{ps}$ pulses (FWHM) with a repetition rate of $714 \mathrm{MHz}$. The electrons are prebunched in two $714 \mathrm{MHz}$ subharmonic bunchers and then bunched and accelerated in an S-band traveling wave buncher, an S-band capture section, and a $2 \mathrm{GeV}$ S-band linac.

Because the NLC design requires relatively low single bunch charge, the important design issues relate to the long bunch trains. Compensation techniques have been devised to control the transient beam loading and the long- 


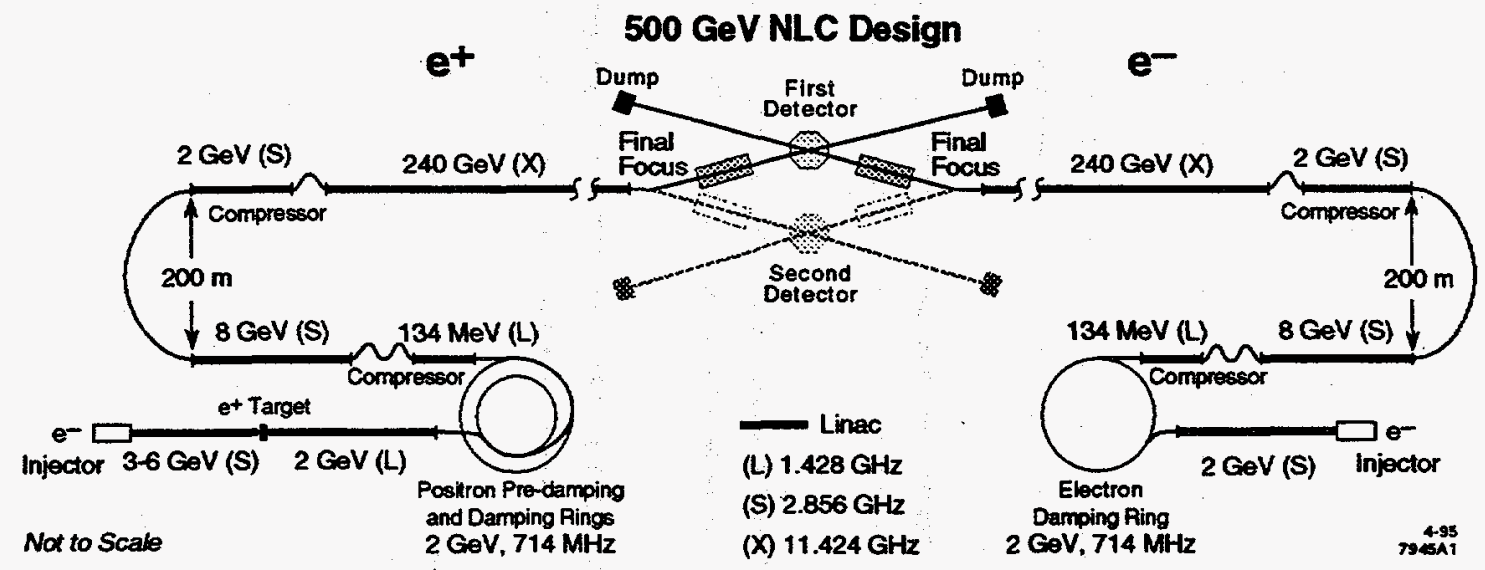

Fig. 1 Schematic layout of the SLAC NLC design.

range transverse wakefields, which are important in the Sband sections, are reduced by using scaled versions of the Damped-Detuned Structures discussed in Sect. IV.

The design of the NLC positron source [4] is also based on its SLC counterpart. It is a conventional source which uses an electromagnetic shower generated by colliding a high energy (3 6 GeV) electron beam with a rotating target. Like the SLC design, the target is followed by a flux concentrator and a capture accelerator embedded in a DC solenoid. To reduce the single pulse heating, the beam size at the target is twice that at the SLC target. Because the larger beam size leads to a larger positron emittance and longer bunch length, the NLC design uses L-band (1428 $\mathrm{MHz}$ ) accelerator structures; the aperture limited emittance is then $\gamma \epsilon_{x, y}=0.06 \mathrm{~m}$-rad.

\section{DAMPING RINGS AND COMPRESSORS}

The damping rings for the NLC [5][6] must produce beams with normalized emittances of $\gamma \epsilon_{x}=3 \times 10^{-6} \mathrm{~m}$-rad and $\gamma \epsilon_{y}=3 \times 10^{-8} \mathrm{~m}$-rad. A single damping ring is used to damp the electron beams. It is 220 meters in circumference and damps four trains of 90 bunches simultaneously; the trains are separated by $60 \mathrm{~ns}$, allowing fast kickers to inject and extract individual bunch trains without disturbing the others.

Because the incoming positrons have a much larger emittance, an additional pre-damping ring is used to damp the $e^{+}$beam. The pre-damping ring is half the circumference of the main damping ring and stores two bunch trains at once. It is a relatively simple ring with a large aperture and a large equilibrium emittance. After the pre-damping ring, the positrons are injected into a main damping ring that is identical to the electron damping ring.

The damping ring designs are similar in many ways to the 3rd generation light sources and can benefit from much of the technology that has been developed. Furthermore, the ATF Damping Ring [7], being constructed at KEK, will experimentally verify many of the design concepts.

After the damping rings, the bunch length must be compressed by a factor of 40 . This is done in two stages [8]. The first stage, located after the rings at $2 \mathrm{GeV}$, compresses the rms bunch length from $4 \mathrm{~mm}$ to $500 \mu \mathrm{m}$. The first stage also contains a spin rotator system, consisting of four solenoids, that provides full control over the orientation of the beam polarization.

Following the first bunch compression, the beam is accelerated to $10 \mathrm{GeV}$ in an S-band linac and then further compressed to a final bunch length of $100 \mu \mathrm{m}$. This second stage compressor is a telescope in longitudinal phase space, preventing energy errors from the pre-linac from becoming phase errors in the X-band linac.

\section{X-BAND RF}

The NLC X-band RF system is based on the SLAC S-band linac RF, but the frequency has been increased to $11.4 \mathrm{GHz}$ to support the higher gradient. The technology to provide the high gradient at high frequency has been under development at SLAC and KEK for the past 8 years. It will be used to provide acceleration at the NLC Test Accelerator (NLCTA) [9] which is presently under construction.

The $500 \mathrm{GeV}$ NLC requires $50 \mathrm{MW} 1.25 \mu$ s klystrons $[10]$ as shown in Table 1 . For economy and efficiency these are planned to be focussed with a periodic permanent magnet (PPM) lattice. Presently there are two klystrons operating at levels exceeding $50 \mathrm{MW}$ with $1.5 \mu$ s pulses. A third klystron has operated at about $60 \mathrm{MW}$ for short pulses and is presently being conditioned for long pulse operation. These three klystrons will be used in the NLCTA to gain operational experience and the NLC PPM klystron is presently undergoing detailed design.

An RF pulse compression system is needed to compress the klystron RF pulse by a factor of 5 while increasing the power by a factor of 3.6. A prototype SLED-II system is presently operating at SLAC. It has achieved pulse compression gain of 4 to 4.4 and has exceeded $200 \mathrm{MW}$ output power. It is presently being used for accelerator structure tests. The three SLED-II systems for the NLCTA are being fabricated.

The accelerator structures for the NLC must control the long-range transverse wakefield to prevent beambreakup while accelerating beams with an unloaded gradient of $50 \sim 100 \mathrm{MV} / \mathrm{m}$. The wakefield is controlled with a Damped-Detuned Structure (DDS) [11] where the transverse modes are both detuned and weakly damped, reducing the Q's to roughly 1000 . A test of a detuned structure (no damping) in the ASSET facility [12] verified the rapid fall 


\section{DISCLAIMER}

This report was prepared as an account of work sponsored by an agency of the United States Government. Neither the United States Government nor any agency thereof, nor any of their employees, makes any warranty, express or implied, or assumes any legal liability or responsibility for the accuracy, completeness, or usefulness of any information, apparatus, product, or process disclosed, or represents that its use would not infringe privately owned rights. Reference herein to any specific commercial product, process, or service by trade name, trademark, manufacturer, or otherwise does not necessarily constitute or imply its endorsement, recommendation, or favoring by the United States Government or any agency thereof. The views and opinions of authors expressed herein do not necessarily state or reflect those of the United States Government or any agency thereof. 


\section{DISCLAIMER}

Portions of this document may be illegible in electronic image products. Images are produced from the best available original document. 
off of the wakefield roughly $1.4 \mathrm{~ns}$ behind the driving bunch; the damping in the DDS structure will further decrease the wake over the long bunch train. This detuned structure has also been tested up to $55 \mathrm{MV} / \mathrm{m}$ and will be conditioned up to about $65 \mathrm{MV} / \mathrm{m}$ with the prototype SLED-II system. Three additional detuned structures for the NLCTA are being brazed. The DDS structure is presently undergoing detailed mechanical design.

\section{X-BAND LINAC}

The X-band linacs [13] accelerate the beams from 10 $\mathrm{GeV}$ to the final beam energy. Each of the linacs includes roughly 700 quadrupoles placed between the accelerator structures in a FODO lattice. To preserve the low emittance beams, very tight tolerances are required on the alignment and RF control. Beam-based techniques are needed to achieve the alignment tolerances. To this end, dipole mode detectors are used in the structures and BPM's are placed in the quadrupoles. In addition, both the structures and the quadrupoles are supported on separate mechanical movers.

Many of the required beam-based alignment techniques are being verified in the SLC. Further tests will be made using ASSET and the NLCTA. While the alignment concepts are straightforward, experimental verification is necessary to understand the practical limitations and long term stability.

\section{COLLIMATION AND FINAL FOCUS}

After the linac and subsequent diagnostics, the beam enters a collimation system [14] which collimates both phases in the horizontal and vertical planes as well as the energy deviation. Although the collimation section is relatively long ( $1.8 \mathrm{~km}$ for the $1 \mathrm{TeV}$ design), it is felt necessary to prevent backgrounds that could overwhelm the detectors.

Following the collimation section, an IP-switch and short arc provide a $10 \mathrm{mrad}$ deflection and direct the beam to one of the two IP's. The design includes two IP's to allow the alternate detector designs and final focus systems that would be required to optimize for $\gamma-\gamma$ and $\gamma-e^{-}$collisions as well as $e^{+}-e^{-}$collisions.

Finally, the beam enters the final focus [15]. At the beginning of the final focus, there are coupling control and beta-matching sections along with phase space diagnostics. The remainder of the final focus optics is similar to the Final Focus Test Beam (FFTB) [16] with the addition of two sextupoles [17] to increase the bandwidth and a crab cavity which is needed due to $10 \mathrm{mrad}$ crossing angle. The system parameters were optimized as described in Ref. [18] and the tolerances are described in Ref. [15]. Based on the SLC and FFTB experience, extensive consideration is being given to the tuning techniques and diagnostics requirements, as well as stability issues. Finally, the beam line from the IP to the dump [19] also contains extensive diagnostics to measure the beam centroid, polarization, and disruption, as well as secondary pairs and beamstrahlung.

\section{DISCUSSION}

In this paper, we have given the primary parameters and described the layout of the SLAC NLC. We have also described the upgrade path to $1 \mathrm{TeV}$, which is being explicitly designed into the collider, and possible upgrade paths to $1.5 \mathrm{TeV}$. Finally, we are designing the collider to operate over a large range of beam parameters to both ensure the feasibility of the upgrades as well as provide operating flexibility. More detailed descriptions of the subsystems and tolerances can be found in the references.

Much of the design is based on the operating experience with the SLC. In addition, many of the novel components of the collider are being or will be tested in specially designed test facilities. In particular, ASSET and the NLCTA will verify the $R F$ system and accelerating structures, the FFTB is studying the final focus designs, and the KEK ATF will study issues for the damping rings.

\section{References}

[1] Proc. of the 1995 Workshop on Next Generation Linear Colliders Tsukuba, Japan, April, 1995.

[2] International Inter-Laboratory Collaboration for Linear Colliders - Technical Review Committee Report, in preparation.

[3] R. Alley, et. al., "The Stanford Linear Accelerator Polarized Electron Source," Submitted to Nucl. Instr. Meth. (1995).

[4] H. Tang, et. al., "The NLC Positron Source," these proceedings.

[5] T. O. Raubenheimer, et. al., "A Damping Ring Design for the SLAC NLC," these proceedings.

[6] T. O. Raubenheimer, et. al., "Collective Effects in the NLC Damping Rings," these proceedings.

[7] J. Urakawa, et. al., Proc. of the 15th Int. Conf. on High Energy Acc., Hamburg, Germany (1992) 124.

[8] P. Emma, et. al., "A Bunch Compressor Design for the Next Linear Collider," these proceedings.

[9] R. D. Ruth, et. al., Proc. of the 1999 Part. Acc. Conf., Washington, D.C. (1993) 543.

[10] G. Caryotakis, et. al., Proc. of the 4th Euro. Part. Acc. Conf., London, England (1994) 1921.

[11] K. Ko, et. al., "Design Parameters for the Damped Detuned Accelerating Structure," these proceedings.

[12] C. Adolphsen, et. al., Phys. Rev. Let. Vol. 74, No. 13 (1995) 2475.

[13] C. Adolphsen, et. al. "Emittance and Energy Control in the NLC Main Linacs," these proceedings.

[14] J. Irwin, et. al., Proc. of the 1993 Part. Acc. Conf. Washington, D.C. (1993) 185.

[15] F. Zimmermann, et. al., "A Final Focus System for the SLAC Next Linear Collider," these proceedings.

[16] K. Oide, "Results of the Final Focus Test Beam," these proceedings.

[17] R. Brinkmann, DESY-M-90-14 (1990).

[18] F. Zimmermann, et. al., "Optimization of the NLC Final Focus System," these proceedings.

[19] J. Spencer, et. al., "The SLAC NLC Extraction and Diagnostic Line," these proceedings. 